\title{
AN INEQUALITY FOR THE TAKAGI FUNCTION
}

\section{ZOLTÁN BOROS}

Abstract. The well-known Takagi function $T(x)=\sum_{k=0}^{\infty} 2^{-k} \operatorname{dist}(x, \mathbb{Z})$ plays a crucial role in the theory of approximately convex functions. In order to establish the sharpness of some Bernstein-Doetsch type results for approximate convexity, we prove that the Takagi function fulfils the inequality

$$
T\left(\frac{x+y}{2}\right) \leqslant \frac{1}{2}(T(x)+T(y)+|x-y|)
$$

for all real numbers $x$ and $y$.

Mathematics subject classification (2000): 26A51, 26A30, 39B62.

Key words and phrases: Approximately Jensen-convex functions; Takagi function.

\section{REFERENCES}

[1] P. BILLingSLEY, Van der Waerden's continuous nowhere differentiable function, Amer. Math. Monthly 89 (1982), 691.

[2] F. S. CATER, On van der Waerden's nowhere differentiable function, Amer. Math. Monthly 91 (1984), 307-308.

[3] G. FABER, Einfaches Beispiel einer stetigen nirgends differentiierbaren Funktion, Jahresbericht der Deutschen Mathematiker-Vereinigung 16/1 (1907), 538-540.

[4] A. HÁZY AND Zs. PÁLES, On approximately midconvex functions, Bull. London Math. Soc. 36 (2004), 339-350.

[5] A. HÁZY AND Zs. PÁLES, On approximately $t$-convex functions, Publ. Math. Debrecen 66/3-4 (2005), 489-501.

[6] H.-H. KaIRIES, Takagi's function and its functional equations, Wyż. Szkoła Ped. Kraków. Rocznik Nauk.-Dydakt. Prace Mat. 15 (1998), 73-83.

[7] K. KNOPP, Ein einfaches Verfahren zur Bildung stetiger nirgends differenzierbarer Funktionen, Math. Z. 2 (1918), 1-26.

[8] Zs. PÁLES, 7. Problem in Report of Meeting, The Forty-first International Symposium on Functional Equations, Aequationes Math. 67 (2004), 307.

[9] T. TAKAGI, A simple example of the continuous function without derivative, J. Phys. Math. Soc. Japan 1 (1903), 176-177.

[10] B. L. VAN DER WAERDEN, Ein einfaches Beispiel einer nichtdifferenzierbaren stetigen Funktion, Math. Z. 32 (1930), 474-475. 